\title{
Bio-Inspired Design of Lightweight and Protective Structures
}

\author{
Prasad S. Mehta, Jennifer Solis Ocampo, Andres Tovar, and Prathamesh Chaudhari \\ Indiana University-Purdue University Indianapolis
}

This is the authors' manuscript of the work published in final form as:

Mehta, P. S., Solis Ocampo, J., Tovar, A., \& Chaudhari, P. (2016). Bio-Inspired Design of Lightweight and Protective

Structures. Presented at the SAE 2016 World Congress and Exhibition. https://doi.org/10.4271/2016-01-0396

\begin{abstract}
Biologically inspired designs have become evident and proved to be innovative and efficacious throughout the history. This paper introduces a bio-inspired design of protective structures that is lightweight and provides outstanding crashworthiness indicators. In the proposed approach, the protective function of the vehicle structure is matched to the protective capabilities of natural structures such as the fruit peel (e.g., pomelo), abdominal armors (e.g., mantis shrimp), bones (e.g., ribcage and woodpecker skull), as well as other natural protective structures with analogous protective functions include skin and cartilage as well as hooves, antlers, and horns, which are tough, resilient, lightweight, and functionally adapted to withstand repetitive high-energy impact loads. This paper illustrates a methodology to integrate designs inspired by nature, Topology optimization, and conventional modeling tools. Two designs are explained to support this methodology: Helmet design inspired by human bone cellular structure (trabecular structure) and vehicle body inspired by a water droplet, ribcage, and human bone. In the helmet design, a finite part of is optimized using topology optimization to generate the porous structure. In the vehicle body design, a water droplet framework, the bio-inspired simulation-based design algorithm used in this work generates innovative layouts. At the vehicle scale, the generated spaceframe has a structure similar to the one of a long bone. In essence, the aerodynamic water droplet shape is protected by the specialized ribcage. At the component scale, each spaceframe tubular component is filled with a functionally graded cellular structure. This internal cellular structure reminds the one of a bone. The spaceframe is attainable with few parts of greater complexity. Such complex, lightweight, multiscale structural layout can be manufactured using $3 \mathrm{D}$ printing technologies.
\end{abstract}

\section{Introduction}

Consistent efforts have been made to design impact-protective structures for several engineering applications using bio-inspired approaches. For instance, scales of animals like fish, crocodile, and turtle have inspired the design of flexible armors [1] . Nature has given some excellent examples that have capabilities of absorbing energy and reducing crushing force. The woodpecker beak and skull are protected from injury despite the repeated impacts due to its internal structure and a spongy hyoid bone that acts as a shock absorber [2]. The internal bone structure within humans is unique which has bone density distributed according to forces acting on it. Bones has a porous structure that is designed to absorb impacts.

Looking at the above examples, today humans are also facing similar problems like to have an impact resistant structure and to have a protective structure to prevent accidents. By studying this 'nature design' or 'bio designs' and implementing it to our engineering design, we can make designs more efficient, lightweight, cost effective and most importantly safe structures.

Bio-inspired design (BID) is emerging in various fields and getting popular. Though there are some methodologies proposed but there is no fixed process to apply these biologically inspired designs for protective structures. There have been several tools and methods proposed to support bio-inspired design []ㅡ, including online repositories such as AskNature from the Biomimicry Institute (www. asknature.org). This repository provides a systematic approach in identifying the functions and properties that can be implemented in mechanical design with the help of available computational tools and database. There have been many efforts done to accomplish light and safe structures. However, very few designs are found to be related to bio-inspired design for protective structures. Implementation of bio designs can be done by scanning all biological substances occurring in the nature that uses some technique to protect themselves. These will solely depend on the purpose of design. Integrating the bioinspired designs and the conventional design can result in better designs. This paper defines the methodology used to implement the bio-inspired design. As the aim of the study is to design protective (crashworthy) structures, similar naturally occurring animals and phenomenon were studied. The computer-aided design (CAD) software was used to design an initial model. These designs were optimized using topology optimization tools. 
This study includes two examples; the protective layer of the helmet is designed which is inspired by the trabecular bone structure, and a vehicle body design is inspired by water droplet for its aerodynamic shape, a human rib cage that acts as a protective structure and bone that absorbs the impact loads. The scaled model are 3D printed which demonstrates the design manufacturability.

\section{Biological Energy Absorbing Structures}

The BID process followed in this work is set on several logical steps with the aim to relate the contributions from engineering (design) and biology (knowledge). From engineering, the design process starts with the definition of the problem, the establishment of requirements and constraints, evolving to the concept generation and continuing through the life cycle of the product or system. From biology, a knowledge-driven approach that starts with discovering nature and the efforts are focused on explaining the phenomena. BID follows the steps of a technical design and to abstract the solutions from nature for the similar problems.

In this study, the problem revolves around improving shock resistant structures, in areas such as energy dissipation and reducing weight; therefore, the desired characteristics should be associated with keywords that help the search among the available tools. The keywords used to explore nature on AskNature include crashworthiness, impact resistance, and lightweight. The online repository comprises a complete catalog of phenomena in nature, the designers must be able to not only look for familiar or obvious solutions but also observe the physical conditions and phenomena under which the state of substance occur. The search results on these tools gave us the information of 15 beings, which are exposed to large instantaneous forces. One of the most significant results is to observe that in all cases, nature solves the impact strength with light weight structures because a greater weight has a direct effect on energy consumption of the species. Additionally, it is concluded that much of the beings combine several of the mechanisms identified to dissipate the impact energy. Subsequently, we abstract and synthesize the mechanisms used by nature to resist shocks. Three common shock-absorbing mechanisms in nature are porous structure and damping, airbags and cushion, and organic circular or spherical shapes.

\section{Porous Structure and Damping}

Bones, beaks of several birds, horns and the peel of some fruits, have high impact strength due to its porous internal structure. This structure provides a structural flexibility in the configuration of the element, beyond the material properties. Overall, the structures are sandwich shaped, where the two outer surfaces have a higher density, and this decreases as it approaches the center of the part. Each time the element is impacted, the porous structure dissipates energy through the deflection of the small cells. Furthermore, the cells are connected to adjacent cells so that the forces are distributed and shared among different elements.

Among the most representative example is the pomelo fruit. The citrus fruit is a native of South America and can withstand falls of up to 10 meters high and to preserve its integrity [4]. Figure 1 shows the internal structure, as mentioned above, its shell has a density gradient along the peel.

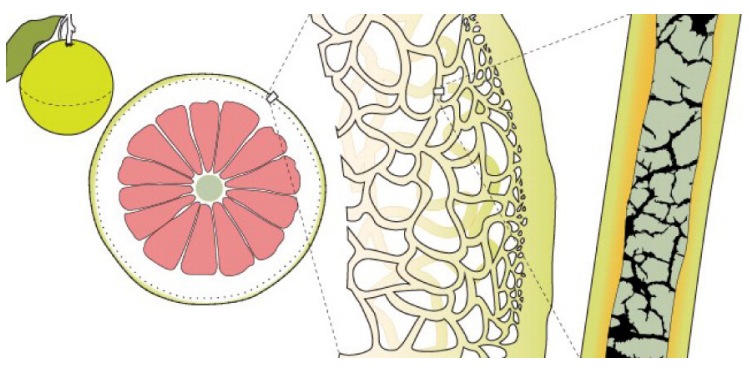

Figure 1. Pomelo fruit pours structure []]

The toucan has a porous internal structure at its beak

Figure 2. The beak is subjected to large forces and even though it is one-third the length of the bird, contributes only to the twenty percent of the weight []ㅡ.

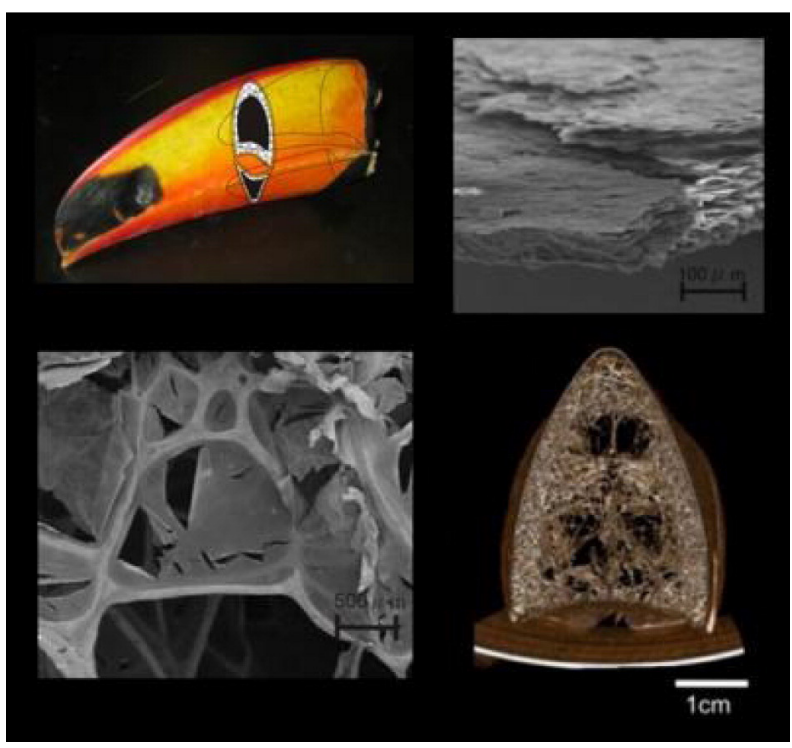

Figure 2. Toucan beak porous structure (Source: AskNature.com)

\section{Airbags and Cushion}

This mechanism corresponds to the use of compressible fluids, or that may move to dissipate energy from the impact. This mechanism can be seen in the form of airbags in various birds Figure 3 . These bags cover the vital organs and play no direct role in the exchange of oxygen and carbon dioxide. It helps to dissipate the extra heat generated during the flight for the birds while diving into the water contributing to absorb the impacts; this is the case of the Pelican [7].

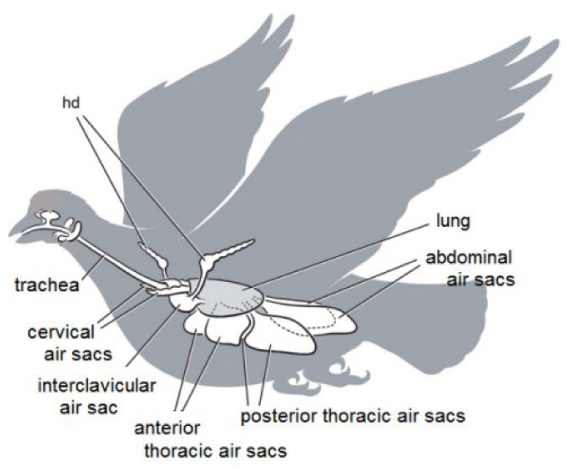

Figure 3. Air sacs in birds [] $]$ 


\section{Organic Circular or Spherical Shapes}

This mechanism, as the name implies, corresponds to the spherical or circular elements found in nature. The spherical shape can provide a better strength and stiffness for given amount of material.
This trend is observed in some fruits like coconut, pomelo fruit and in some sea urchins, called 'tests' which must withstand the constant force of the waves.

\section{BID Methodology for Protective Structures}

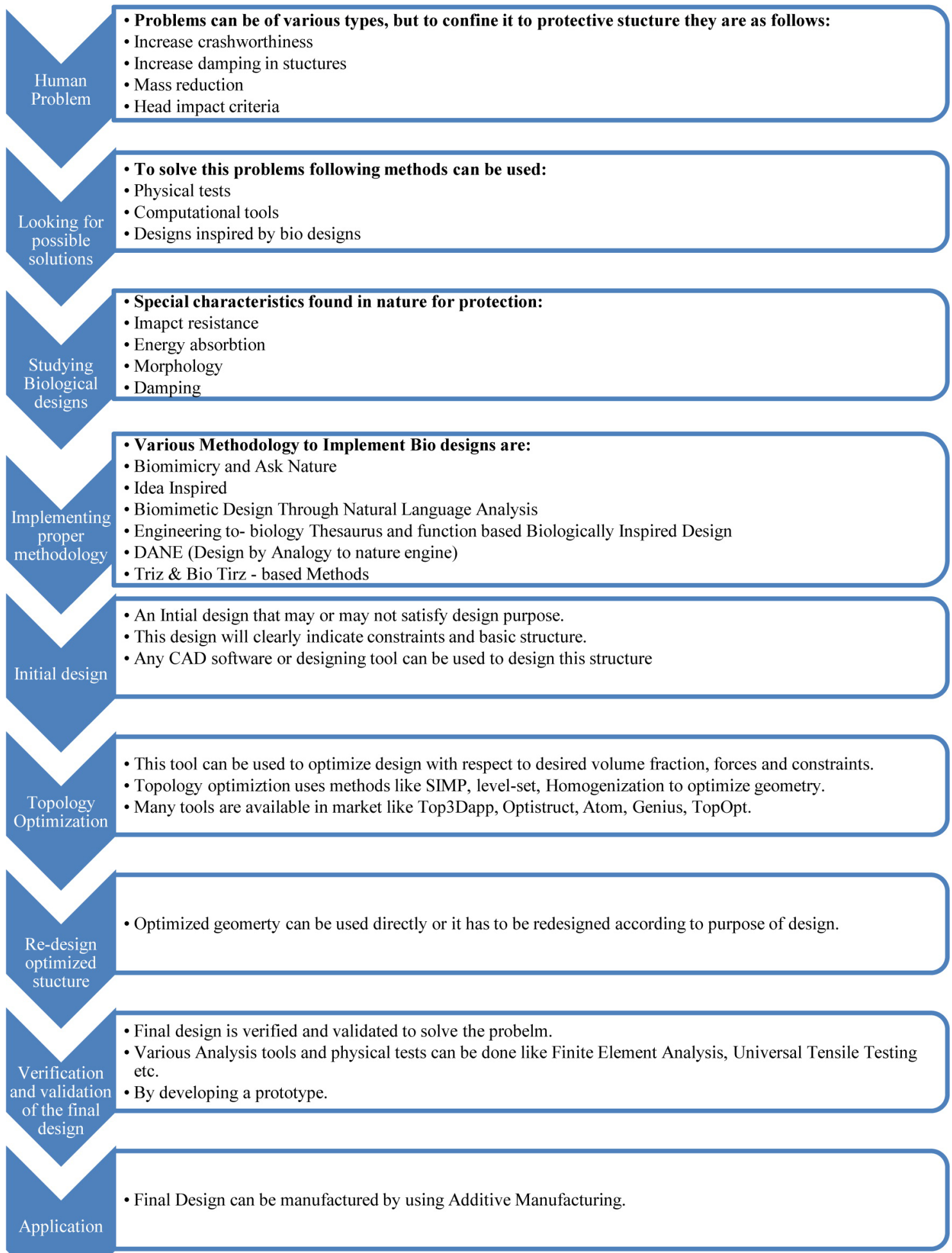




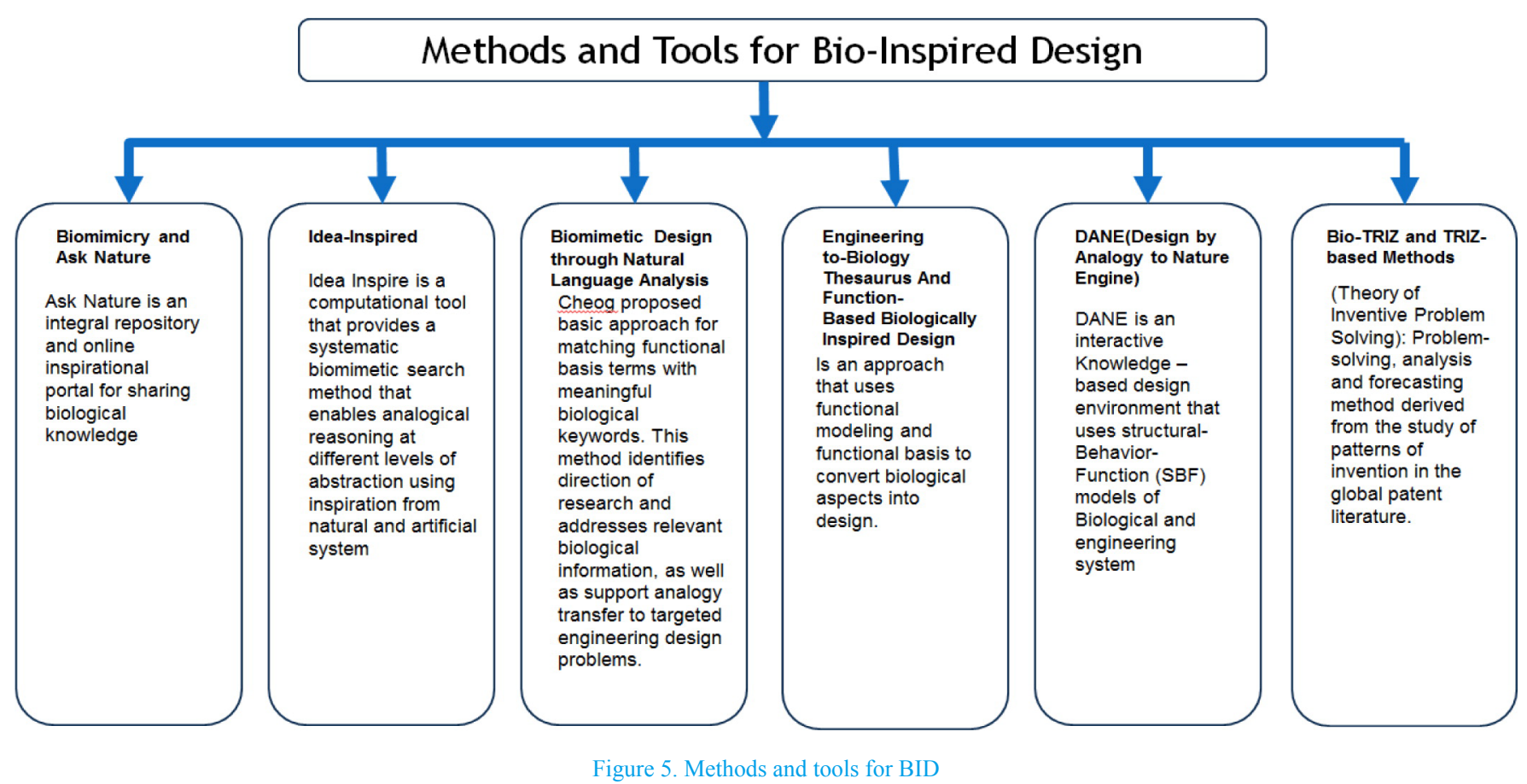

Once the study of the bio designs is completed, an initial design can be developed based on mechanisms, loads, and constraints. This design essentially will be based upon bio designs. Figure 5 describes the summary of various methods and tools to implement bio designs to engineering designs [ $\underline{3}]$. These methodologies give us a pathway to relate human problems to bio designs. Now we have an initial design ready that identifies the main mechanisms through which nature solves the problem.

So far, the decision has been based on observation and information collected about the behavior of natural systems. At this point, we incorporate a new tool, which has been fundamental to our design and development process. In order to guide the design and provide a clear picture of stress conditions, the initial design previously developed is used to run an analysis of topology optimization. The results of this approach are harmonious with the mechanisms that can be observed in nature through the evolution of thousands of years, as both seek to optimize designs and minimize unnecessary items. Thus, nature allows us to identify the shape and configuration of the elements that resist impact loads. The Matlab-based iterative topology optimization algorithm is implemented to perform the analysis []]. Further, designs can be tested through modeling, simulation or construction of functional prototypes. The test methods are at the discretion of the designer according to the criticality of the item.

The design inspired by nature provides a starting point to look for innovative and optimal solutions based on years of evolution. By paying attention to the trends of the solutions proposed by nature gives us a notion of the optimization of the structures. A new methodology of integrating bio designs with topology optimization is illustrated in a schematic representation with methods and tools in Figure 4.

\section{Design of Engineering Protective Structures}

Following the defined BID approach, the two examples of BIDs for crashworthiness and light structure are presented: helmet design and vehicle body design.

\section{Helmet Design}

\section{Introduction}

Helmets have proved to protect and reduce head injuries caused by accidents on motorcycles/ bicycles [9]. The key objective of the helmet is to protect the head from tangential impacts that would transfer large forces to head [10]. The helmet is made up of three layers, outer, protective and comfort layer. Protective layer acts as impact absorbent [11]. "When impacted, the rigid outer shell structure serves to increase the initial contact area, thereby decreasing the compressive stress exerted on the underlying EPS liner and so enhancing energy dissipation through crushing. This two-phase process of energy dissipation attempts to reduce the incidence of cranial fractures (by reducing localized strain on the skull) and brain injury (through managing the translational acceleration of the head)" [10].

Although today advancement in helmet design has resulted in developing a crashworthy helmet, still there is wide scope for development. The designed helmets are effective in managing translational accelerations while they lack in managing rotational acceleration [12-13]. Additionally, improper fit of the helmet may not absorb the impact forces as desired [14-15]. Therefore, it becomes essential to investigate new ways to design helmets for safety. The new designing and manufacturing tools give us the advantage to develop a complex structure that can attenuate energy effectively. Cellular structures can be used to design a complex helmet structure. The cellular structures are capable of dissipating the energy. Human bone is a good example for attenuating energy due to its porous nature. Also, porous structures like honeycombs and foams pose the ability to dissipate energy by deformation [16] .

Topology optimization is an optimal material distribution within a given design domain, for a set of given loads and boundary conditions. Top3Dapp is used to perform the topology optimization []. Top3Dapp solves the compliance problem by optimizing given design domain that results in the stiffest structure. Solid Isotropic Material with Penalization (SIMP) method is used in code to solve the optimization problem. SIMP method implements power-law 
interpolation function between void and solid in given design domain. The power law penalizes the intermediate density values that drives the design towards a black-and-white structure [17]. This method is based on a heuristic relation between element density xi and element Young's modulus Ei given by following equations,

$$
\begin{array}{lr}
E_{i}\left(x_{i}\right)=x_{i}^{p} E_{0} & (p \geq 1) \\
\rho_{i}\left(x_{i}\right)=x_{i}\left(\rho_{0}\right) & \left(0 \leq x_{i} \leq 1\right)
\end{array}
$$

Where $\mathrm{p}$ is penalization power, $E_{0}$ is the elastic modulus of base material $\rho_{0}$ and $\rho_{t}$ is variable density.

A unique approach is implemented to design the helmet. The stiff mesostructure is generated using topology optimization and further imbibed in a macrostructure i.e. protective layer of the helmet. The resultant structure is comparable to that of trabecular structure found in the bone.

\section{Methods}

The method to design helmet is divided into three stages: a) Initial design of helmet and voxelization. b) Extracting a finite part and performing topology optimization. c) Imbibing mesostructure into the macrostructure.

\section{The Initial Design of Helmet and Voxelization}

The Initial design of protective layer was designed using SolidWorks software and design was exported in STL file format. The Solidworks model replicates the shape of a protective middle layer of the helmet. The design was imported in Matlab and voxelized using voxelization code (Figure 6). Voxelization is done to convert STL file into a 3D matrix that is made up of 1 and 0 digits. Where 1 represents material and 0 represents void. During the process of voxelization, a number of voxels in $\mathrm{X}, \mathrm{Y}$ and $\mathrm{Z}$ directions should be defined. In this work, we used 20 elements in X-direction, 20 in Y-direction and 20 in the $\mathrm{Z}$ direction.

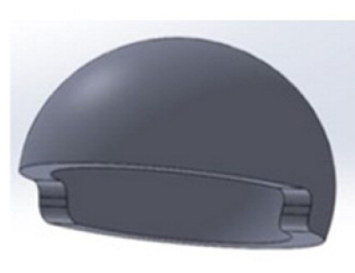

(a)

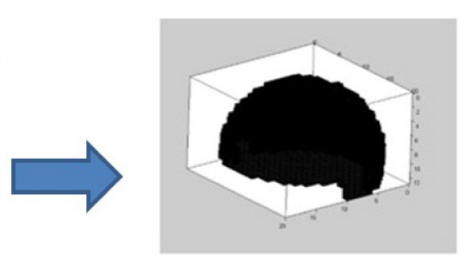

(b)
Figure 6. a) Initial design of protective layer in SolidWorks. b) Voxelized design using Matlab.

\section{Extracting a Finite Part and Performing Topology Optimization}

Further, a finite part of the protective layer was extracted, and topology optimization was carried out on the part. The part was discretized into 10 elements in X-direction, 10 in Y-direction and 10 in the $\mathrm{Z}$ direction. The optimization problem is subject to element material distribution with predetermined volume fraction constraint. The other parameters considered for topology optimization are as shown in Table 1.
Table 1. Parameters of Topology optimization of a part

\begin{tabular}{|l|l|l|}
\hline No & Parameter & Value \\
\hline 1 & Volume fraction & 0.3 \\
\hline 2 & Penalization factor & 3.0 \\
\hline 3 & Filter radius & 1.2 \\
\hline 4 & Element size & $10 * 10^{*} 10$ \\
\hline 5 & $\begin{array}{l}\text { Maximum number of } \\
\text { iterations }\end{array}$ & 200 \\
\hline
\end{tabular}

As the extracted part is relatively small with respect to the structure it is assumed that the force is acting at the center of the part. The coordinates of the load point are: $(5,5,5)$.

The part is supported in all corners. The co-ordinates of the support points are: $(0,0,0) ;(10,0,0) ;(10,0,10) ;(0,0,10) ;(0,10,0) ;(10$, $10,0) ;(10,10,10) ;(0,10,10)$.

Figure 7 shows the extraction of the finite part, supports, forces and the optimized finite part that gives a mesostructure.

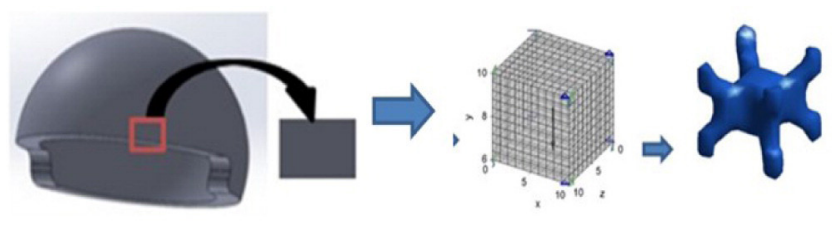

(a)

(b)

Figure 7. a) Finite part of the helmet is extracted to perform topology optimization. b) Top3Dapp is used to optimize the extracted part, and the blue structure in figure resembles trabeculae.

\section{Results: Imbibing Mesostructure into the Macrostructure}

The Matlab code was written to imbibe the optimized structure in voxelized design to get the cellular structure. This cellular helmet structure is saved in Matlab file format (*.mat). This file is used as input in the code of generating STL file in Matlab. The output from Matlab is STL file that can be used for Additive Manufacturing. Figure 8 illustrates the protective layer of the helmet in STL file format. An STL file stores the geometry in the triangular surface mesh format. The data is stored in a $3 \mathrm{D}$ matrix format that contains $\mathrm{X}, \mathrm{Y}$ and $\mathrm{Z}$ coordinates of each node.

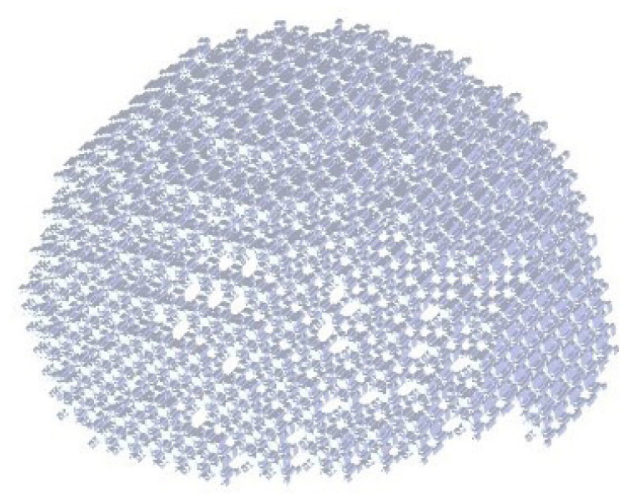

Figure 8. Output from Matlab of protective layer 
As the loads and supports were symmetric, the resultant optimized structure became symmetric. Due to this asymmetric structure, it made possible to imbibe mesostructure into the macrostructure without any alignment constraints.

\section{D Printed Model}

A high-resolution object30 pro printer was used to print this model. Objet30 Pro is ideal for prototyping. The object30 printer uses the technology of Polyjet printing. Polyjet printing is similar to inkjet printing. It has two jets; one for photopolymer resin and the other for support material. The photopolymer used is rigid opaque material veroblue RGD840 and support material is FullCure 705 non-toxic gel-like photopolymer. Resolution of the printer is $0.1 \mathrm{~mm}[\underline{18}]$. Accuracy varies according to geometry, size, and the orientation of the part. The STL file that is the output of Matlab code is directly used as input to the 3D printer. Figure 9 shows the front view and isometric view of printed protective layer of the helmet.

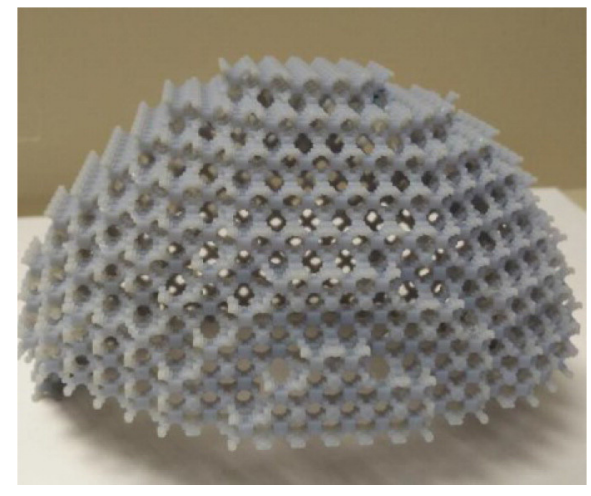

a.

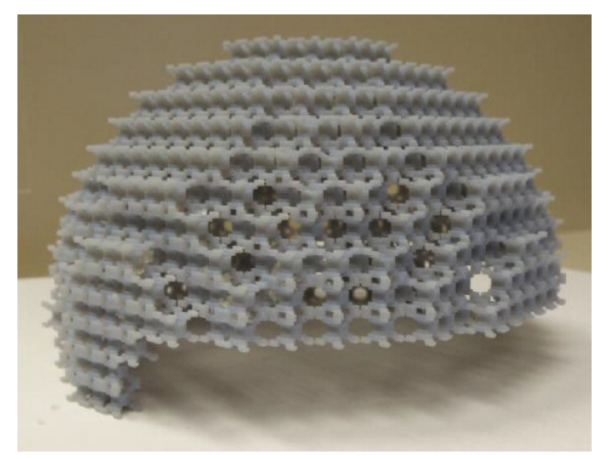

b.

Figure 9. 3D printed helmet protective layer with uniform density (a) Front view, (b) Isometric view.

\section{Vehicle Body Design}

\section{Introduction and Initial Design}

Researchers and automotive industries in the world have been consistent in developing vehicles to make it lightweight, efficient and safe. However, very few researchers have explored nature and tried to integrate it with vehicle design. The proposed vehicle body has two parts; the outer shape (envelope) of a water droplet, and embedded trabecular bone structure (spaceframe). To make a vehicle efficient, it has to be aerodynamic. The shape of a water droplet (blue highlighted part Figure 10) is assumed to be completely aerodynamic in shape as it provides a low drag coefficient. A basic vehicle body was designed by considering the shape of a water drop. The design of the spaceframe provides the mechanical strength and energy absorption capabilities that are required to protect the occupant during a collision event.

The design is the substitute for the traditional metal body with a single structure of a metallic space frame and lightweight polymer composite envelope. The envelope is made up of a polymer composite that will provide desirable safety characteristics as that of a monocoque design found in Formula 1 cars and aircraft. The metallic (functionally graded cellular) spaceframe provides a level of protection similar to race cars that also uses spaceframe or cage design, such as NASCAR cars. These results in very light, strong, and safe design.

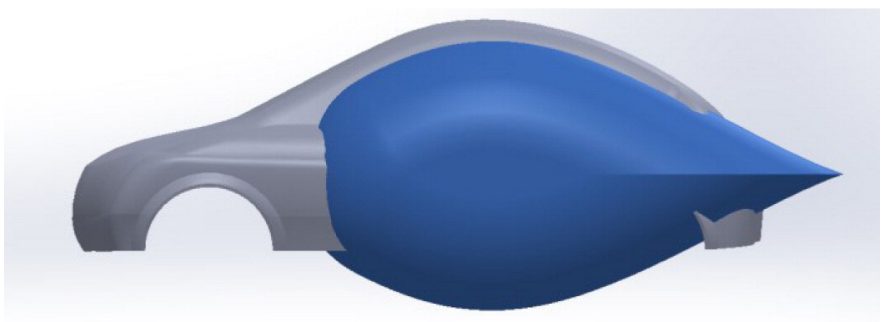

Figure 10. Car representing water-droplet shape

\section{Methods}

\section{Topology Optimization and Crashworthiness}

Topology optimization defines material distribution within the given design domain. The topology optimization algorithm used is referred as Hybrid Cellular Automaton (HCA). "HCA is a methodology developed to stimulate the process of structural adaptation in bones' [19]. The main purpose to use Topology Optimization here is to make vehicle body lightweight and crashworthy. To make any structure crashworthy implies maximization of internal energy absorption. Therefore, objective function is to maximize internal energy (IE) absorption subjected to mass (M), and this optimization will result in material distribution $\mathrm{x}[\underline{20}]$ that is

$$
\begin{aligned}
& \text { Find } \quad \mathrm{x} \in \mathbb{R}^{n} \\
& \text { Maximize } \quad \operatorname{IE}(\mathrm{x})=\int_{0}^{\Delta} \mathrm{P}(\delta, \mathrm{x}) \mathrm{d} \delta \\
& \text { Subject to } \quad \mathrm{M}(\mathrm{x})=\int_{\Omega} \rho(\mathrm{x}) \mathrm{d} \Omega=\text { Mo } \\
& \text { xi } \in\{0,1\}, \quad i=1, \ldots, n
\end{aligned}
$$

Where $\mathrm{P}(\delta, \mathrm{x})$ reaction force after the collision, $\Delta$ is a crushing distance, $\rho(x)$ is material density, M0 is the mass target, and $\Omega$ is the volume of the design domain. The design domain is discretized into $\mathrm{n}$ elements (voxels) so each voxel will have a value of solid when $\mathrm{xi}=$ 1 or void when $\mathrm{xi}=0$.

The loading conditions for the spaceframe two frontal pole impacts (full front and offset) and three side impacts (central and two offset) (Figure 11a) are considered, which satisfies all safety standards. Only half structure was considered for optimization. Supports are applied at the rear, and another symmetric condition is applied at the section of the vehicle body. The volume fraction considered for optimization is 0.5 of defined design domain. The optimized half body was mirrored. Figure 11b illustrates final optimized design. 


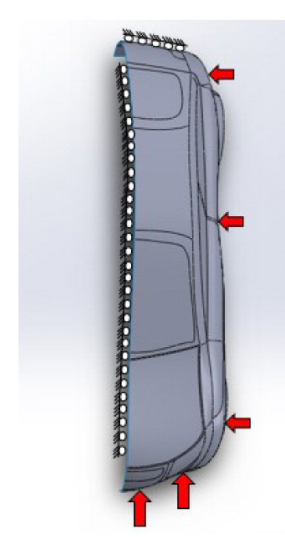

a.

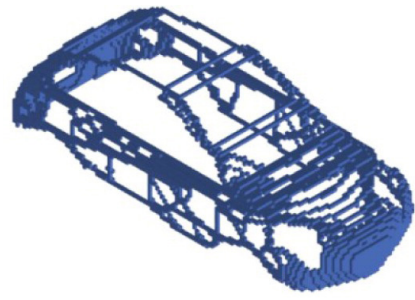

b.
Figure 11. a) Loading conditions applied to the vehicle body (b) Vehicle body output using topology optimization tool (Credit: Kai Liu)

\section{Bone Function Adaptation}

The primary role of the human rib cage is to protect vital organ that lies inside ribs. This methodology was implemented to protect the passengers by generating the structure of spaceframe.

The spaceframe is designed based on results of optimized design from topology optimization tool. The internal structure of each spaceframe tube is filled with functionally graded materials, so the structure can manage the impact with minimum weight without scarifying crashworthiness. The spaceframe was designed using CAD software SolidWorks. The envelope and spaceframe are assembled to make a single structure as shown in Figure 12b.

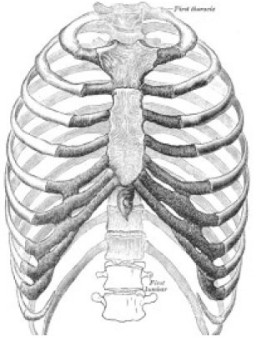

(a)

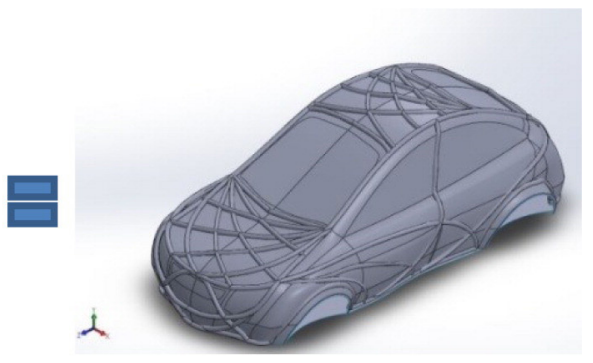

(b)
Figure 12. (a) Human rib cage (Source: Wikipedia, Rib Cage). (b) Structure mimicking human rib cage

\section{Manufacturing of Vehicle Body}

$3 \mathrm{D}$ printing technology made possible to manufacture multi-scale and multi-material model. Taking advantage of this technology vehicle body uses two different materials; ABS and ALSi10Mg alloy. The material for the envelope is ABS and ALSi10Mg is for spaceframe. The $3 \mathrm{D}$ printing is suitable for manufacturing complex structure as that of designed vehicle body. The vehicle body will be 3D printed in two stages; Initially, the functionally graded aluminum alloy spaceframe will be printed using powder sintering or direct metal printing process. Further, using big area additive manufacturing (BAAM) ABS will be printed.

\section{Conclusion}

In this study, we have demonstrated a different approach towards the design of protective structures. The unique results that were obtained after the rigorous study of bio-designs and scrupulous implementation of topology optimization will potentially help in assuaging a serious human problem of vehicular safety. The previous gap of CAD software's which doesn't support the design of porous structures was filled here by successfully implementing Matlab code. As volume fraction was 0.3 for mesostructure, certainly it made helmet lighter by $70 \%$. The design of the helmet and vehicle body illustrates the methodology to implement bio-designs with topology optimization and traditional CAD modeling. The transitional and rotational accelerations in the helmet can be calculated using finite element analysis. The results can be incorporated to modify the current cellular helmet design. In vehicle body design, the water drop shape has low drag coefficient hence increases fuel efficiency. The spaceframe of the vehicle is generated by topology optimization to increase energy absorption and to minimize the penetration during a crash event. The functionally graded spaceframe manages the kinetic energy with minimum weight and without scarifying crashworthiness. The crashworthiness of a vehicle design must be verified with numerical and physical tests. Usually, component tests, sled impact test, and full-scale barrier impact test are the tests carried out to verify crashworthiness (according to FMVSS regulations). These tests are not considered yet to validate these designs. In the end, we have conceived a functioning methodology that could be emulated in further studies of bio-inspired designs. The defined methodology can be used to generate various cellular structures and designs such as vehicular structures, ballistic and other protective structures.

\section{References}

1. Chintapalli, R.K., et al., Fabrication, testing and modeling of a new flexible armor inspired from natural fish scales and osteoderms. Bioinspiration and Biomimetics, 2014. 9(3).

2. Wang, L., et al., Why Do Woodpeckers Resist Head Impact Injury: A Biomechanical Investigation. PLoS ONE, 2011. 6(10): p. e26490.

3. Fu, K., et al., Bio-inspired design: An overview investigating open questions from the broader field of design-by-analogy. Journal of Mechanical Design, Transactions of the ASME, 2014. 136(11).

4. Fischer, S.F., et al. Pummelos as concept generators for biomimetically inspired low weight structures with excellent damping properties. 2010. P.O. Box 101161, Weinheim, D-69451, Germany: Wiley-VCH Verlag.

5. Speck, t. Hierarchical organization of peel confers impact resistance: pomelo.

6. Seki, Y., Schneider M.S., and Meyers M.A., Structure and mechanical behavior of a toucan beak. Acta Materialia, 2005. 53(20): p. 5281-5296.

7. Sereno, P.C., et al., Evidence for Avian Intrathoracic Air Sacs in a New Predatory Dinosaur from Argentina. PLoS ONE, 2008. 3(9): p. e3303.

8. Liu, K. and Tovar A., An efficient 3D topology optimization code is written in Matlab. Structural and Multidisciplinary Optimization, 2014. 50(6): p. 1175-1196. 
9. Liu, B., et al., Helmets for preventing injury in motorcycle riders (Review), in The Cochrane Collaboration. 2009, John Wiley \& Sons, Ltd.

10. BS EN 1078:2012+A1:2012 (2012) Helmets for pedal cyclists and for users of skateboards and roller skates. British Standards Institution (BSI), London

11. Soe S.P.Martin P., Jones M., Robinson M, Theobald P., 'Feasibility of optimizing bicycle helmet design safety through the use of additive manufacturing TPE cellular structure', The International Journal of Advanced Manufacturing Technology, Springer,2015.

12. AareM, Kleiven S, Halldin P. Injury tolerances for oblique impact helmet testing. Int J Crashworthiness. 2004;9:15-23.

13. Iman Ebrahimia, Farid Golnaraghia \& Wanga G. Gar, "Factors Influencing the Oblique Impact Test of Motorcycle Helmets", A School of Mechatronic Systems Engineering, Simon Fraser University, Surrey, British Columbia, Canada.

14. Parkinson Gregory W., MD, FAAP, and Hike Kelly E., BA, "Bicycle Helmet Assessment During Well Visits Reveals Severe Shortcomings in Condition and Fit".

15. Rivara FP, Astley SJ, Clarren SK, Thompson DC, Thompson RS. Fit of bicycle safety helmets and risk of head injuries in children. Inj Prev. 1999;5:194-197

16. Gibson LJ, Ashby, MF (1997) Cellular solids: structure and properties. Cambridge University Press, Cambridge

17. Bendsøe MP, Sigmund O, "Topology optimization: theory, method, and applications", Springer

18. http://www.stratasys.com/3d-printers/design-series/objet30-pro, "objet30 pro Spec sheet".
19. Tovar, A., et al., Topology optimization using a hybrid cellular automation method with local control rules. Journal of Mechanical Design, Transactions of the ASME, 2006. 128(6): p. 1205-1216.

20. Christensen, P.W. and Klarbring A., An introduction to structural optimization. Solid Mechanics and its Applications, 2008. 153: p. $1-220$.

\section{Contact Information}

Andres Tovar, Ph.D. in Aerospace and Mechanical Engineering Phone

Phone no: +1 (317) 278-7090

tovara@iupui.edu

Prasad Mehta, MS in Mechanical Engineering,

Phone no: +1 (317) 603-3662

psmehta@iupui.edu

\section{Acknowledgments}

The authors would like to thank and acknowledge the support of the Advanced Research Projects Agency - Energy - U.S. Department of Energy (ARPA-E) and Local Motors through 2015 LITECAR Challenge as well as Honda R\&D Americas. Any opinions, findings, conclusions, and recommendations expressed in this investigation are those of the writers and do not necessarily reflect the views of the sponsors. The authors would like to thank the participation of Mr. Fabian Lischke, who provided support in the 3D printing of some of the designs and Mr. Kai Liu who provided support for the use and results obtained with top3d. 